\title{
A Systematic Literature Review of Economic Evaluations of Antibiotic Treatments for Clostridium difficile Infection
}

\author{
Hannah E. Burton ${ }^{1} \cdot$ Stephen A. Mitchell ${ }^{1} \cdot$ Maureen Watt $^{2}$
}

Published online: 5 September 2017

(c) The Author(s) 2017. This article is an open access publication

\begin{abstract}
Background and Objective Clostridium difficile infection (CDI) is associated with high management costs, particularly in recurrent cases. Fidaxomicin treatment results in lower recurrence rates than vancomycin and metronidazole, but has higher acquisition costs in Europe and the USA. This systematic literature review summarises economic evaluations (EEs) of fidaxomicin, vancomycin and metronidazole for treatment of CDI.

Methods Electronic databases $\left(\right.$ MEDLINE $^{\circledR}$, Embase, Cochrane Library) and conference proceedings (ISPOR, ECCMID, ICAAC and IDWeek) were searched for publications reporting EEs of fidaxomicin, vancomycin and/or metronidazole in the treatment of CDI. Reference bibliographies of identified manuscripts were also reviewed. Cost-effectiveness was evaluated according to the overall population of patients with CDI, as well as in subgroups with severe CDI or recurrent CDI, or those at higher risk of recurrence or mortality.

Results Overall, 27 relevant EEs, conducted from the perspective of 12 different countries, were identified. Fidaxomicin was cost-effective versus vancomycin and/or metronidazole in 14 of 24 EEs (58.3\%), vancomycin was
\end{abstract}

Electronic supplementary material The online version of this article (doi:10.1007/s40273-017-0540-2) contains supplementary material, which is available to authorized users.

Hannah E. Burton

hburton@teamdrg.com

Maureen Watt

maureen.watt@astellas.com

DRG Abacus, Bicester, UK

2 Astellas Pharma Inc., 2000 Hillswood Drive, Chertsey KT16 ORS, UK cost-effective versus fidaxomicin and/or metronidazole in five of 27 EEs (18.5\%) and metronidazole was cost-effective versus fidaxomicin and/or vancomycin in two of 13 EEs (15.4\%). Fidaxomicin was cost-effective versus vancomycin in most of the EEs evaluating specific patient subgroups. Key cost-effectiveness drivers were cure rate, recurrence rate, time horizon, drug costs and length and cost of hospitalisation.

Conclusions In most EEs, fidaxomicin was demonstrated to be cost-effective versus metronidazole and vancomycin in patients with CDI. These results have relevance to clinical practice, given the high budgetary impact of managing CDI and increasing restrictions on healthcare budgets.

Other This analysis was initiated and funded by Astellas Pharma Inc.

\section{Key Points for Decision Makers}

The cost-effectiveness of fidaxomicin, vancomycin and metronidazole for treating Clostridium difficile infection (CDI) has been evaluated in a number of studies.

The current systematic literature review summarises the results of these economic evaluations, highlighting that despite its higher acquisition cost, fidaxomicin is cost-effective versus metronidazole and vancomycin in these patients.

These results have important implications for clinical practice given increasing restrictions on healthcare budgets. 


\section{Introduction}

The incidence of Clostridium difficile infection (CDI) has increased in the last decade as a result of large outbreaks, resistant strains, severe infection and the spread to new, previously unaffected patient groups without known risk factors [1]. This is despite concerted efforts to improve prevention and curb spread in nosocomial settings [2, 3].

CDI has a substantial impact on patients. It is associated with significant morbidity, hospitalisation and mortality $[4,5]$, which are compounded by the highly recurrent nature of the infection. Approximately $25 \%$ of patients treated with vancomycin or metronidazole, two of the recommended treatment options for CDI in the USA and Europe, experience CDI recurrence [6-8]. In the USA, it has been estimated that CDI recurrence accounts for 75,000-175,000 additional cases per year [3]. Furthermore, patients who have had a recurrence are at increased risk of further episodes [9]; approximately $40-60 \%$ of patients with a first recurrence develop subsequent recurrences [10]. This cycle of infection represents the greatest hurdle for recovery [11] and exacerbates the significant morbidity and economic impact associated with CDI [11, 12].

Managing CDI is costly. In a previously published systematic review of 13 studies, the incremental costs of managing a primary CDI case ranged from US $\$ 2871$ to US $\$ 4846$ in the USA and US\$5243 to US\$8570 outside the USA [13]. Costs include those for medication and laboratory tests [13], but the biggest cost driver is extended hospitalisation. For example, in an EE conducted in the UK, estimated costs per case were $£ 90$ for medication, $£ 87$ for laboratory tests and £2691 for excess hospitalisation; the latter therefore represented $94 \%$ of the total cost [14]. The costs associated with managing recurrent CDI appear to be even greater, reflecting not only re-hospitalisation, but also the need for environmental decontamination, rigorous hygiene in patient care, and in some cases, cohort isolation and ward closure [15]. Indeed, in the systematic review [13], cost estimates for recurrent cases varied from US $\$ 13,655$ to US $\$ 18,067$ in the USA and were estimated to be US\$13,655 outside the USA.

CDI recurrence following treatment with metronidazole or vancomycin is thought to be due to antibiotic-induced disruption of the gut flora and the inability of these agents to prevent the subsequent effects of infection with residual C. difficile spores $[9,16]$. Fidaxomicin is a first-in-class macrocyclic antibiotic for CDI, which combines bactericidal activity against $C$. difficile [17] with limited activity against components of the normal commensal gut flora (compared with vancomycin) [18]. Unlike metronidazole and vancomycin, fidaxomicin also inhibits sporulation of
C. difficile (ATCC 43255 and UK-14 strains) in vitro [16] and it significantly reduced the spore count versus vancomycin in patients being treated for a first episode of CDI [19]. Furthermore, fidaxomicin prevents subsequent recovery of spores, vegetative growth and toxin production even if residual spores remain following treatment [20].

These characteristics of fidaxomicin appear to translate into tangible clinical benefits for patients. In two randomised, double-blind, phase III studies versus vancomycin in patients with CDI, fidaxomicin was associated with statistically significant improvements in recurrence rates and, hence, sustained cure rates [21, 22]. A recent meta-analysis of the data from these studies confirmed these statistically significant improvements, and similar results were obtained in subgroup analyses of patients with severe and non-severe CDI [23]. Furthermore, current evidence shows that the superiority of fidaxomicin over vancomycin is maintained in groups known to be at high risk of recurrence (e.g. in patients with a previous recurrence, those on concomitant antibiotic therapy, those aged $>65$ years, those with cancer, or those with chronic renal insufficiency) [24]. There are no direct comparative studies of fidaxomicin versus metronidazole, but according to indirect treatment comparisons using vancomycin as the data bridge, fidaxomicin was associated with improved sustained cure rates versus metronidazole in the overall CDI patient population [23], as well as in various patient subgroups: patients aged $\geq 65$ years, those with an initial episode, those on antibiotics, or those with severe or nonsevere CDI [25].

The acquisition cost of a course of fidaxomicin is substantially higher than that for metronidazole or vancomycin in Europe and the USA [26-29], which may limit first-line use in some settings. However, given the clinical benefits of fidaxomicin compared with other antibiotics, it is pragmatic to look beyond acquisition costs using economic evaluations (EEs). This is especially important given the high healthcare resource costs associated with managing recurrent CDI [15]. The aim of the current systematic literature review was therefore to summarise published EEs that have assessed the cost-effectiveness of first-line agents, fidaxomicin, vancomycin and/or metronidazole, for treatment of CDI.

\section{Methods}

The methodology used to conduct the systematic review complied with guidance from the Centre for Reviews and Dissemination [30] and the Cochrane Collaboration [31]; however, grey literature was not included in the searches. 


\subsection{Electronic Database Search}

The following electronic databases were searched via the OVID platform on 18 August 2016:

MEDLINE $^{\circledR}$ In-Process and Other Non-Indexed Citations; MEDLINE ${ }^{\circledR}$; Embase; and the Cochrane Library, incorporating the Health Technology Assessment Database and the NHS Economic Evaluation Database. Search terms used to identify relevant EEs are provided in the electronic supplementary material (ESM), online resource 1, Supplementary Table 1.

To be eligible for inclusion in the analysis, EEs had to meet pre-specified inclusion and exclusion criteria (see the ESM, Supplementary Table 2). Any cost-utility, cost-effectiveness, cost-benefit or cost-minimisation analyses that evaluated first-line antibiotic treatments (fidaxomicin, vancomycin and/or metronidazole) for CDI were eligible for inclusion. There were no restrictions in terms of the age of patients or the setting in which they were treated, the antibiotic dose or treatment regimen, the countries of interest, the language of the publication, or the publication date. Publications identified using the electronic database search were independently reviewed for relevance by two of the authors (HB and SM) on the basis of title and abstract. Full publications of potentially relevant citations were then obtained and examined in detail against the eligibility criteria. Disputes regarding eligibility were resolved through discussion between HB and SM. Reasons for exclusion were documented for all excluded citations. Analyses comparing faecal microbiota transplant (FMT) with only one intervention of interest were excluded, as FMT is only listed by guidelines for multiple recurrences (i.e. it is not a first-line treatment) [32-34] and is conducted by only a minority of hospitals in Europe. However, analyses comparing FMT with two or more first-line treatments were included so that data from the comparison of interest (e.g. vancomycin versus metronidazole) could be used.

The reference lists of eligible publications were also reviewed to identify any further potentially relevant publications that were not identified during the electronic database searches.

\subsection{Conference Proceedings}

In order to comply with Cochrane Collaboration guidelines [31], relevant data from conference proceedings were also included. Thus, proceedings from the following conferences, which took place between 2013 and August 2016, were searched: International Society for Pharmacoeconomics and Outcomes Research (ISPOR)-European and USA meetings; European Congress of Clinical Microbiology and Infectious Disease (ECCMID); Interscience
Conference on Antimicrobial Agents and Chemotherapy (ICAAC); and IDWeek. A full list of the conferences included in the search is provided in Supplementary Table 3 (see the ESM).

The following search terms were used to identify relevant abstracts and posters within the conference proceedings: 'difficile', 'clostridium' and 'CDI'. Conference websites were searched to identify posters associated with published abstracts. If the poster was not available online, an attempt was made to contact the authors to obtain a copy.

As data presented at scientific conferences do not undergo the same rigorous peer review as those in full publications (and do not provide the same level of detail), a summary of the results excluding conference presentations was also conducted.

\subsection{Data Extraction}

Key details from included EEs were extracted by HB and verified by SM; these included details of the following: the patient population [diagnosis (e.g. initial episode, recurrent $\mathrm{CDI}$ ), age, subgroups analysed]; interventions for CDI; the model used; sources of clinical, cost and utility data; the results [e.g. the incremental cost-effectiveness ratio, cost per outcome avoided, willingness-to-pay (WTP) threshold]; and study conclusions. Any disputes were resolved through discussion. If the data were published as both abstract or poster and full publication, data were extracted from the latter. If more than one poster was available for a specific EE, the poster containing the most relevant data was used.

As well as cost-effectiveness results for the overall population with CDI, data for specific patient subgroups were also extracted, if reported. Subgroups of interest, which were guided by those reported in the pivotal phase III trials comparing fidaxomicin with vancomycin [21, 22], included patients with recurrent CDI, those at increased risk of mortality or recurrence, and those with severe CDI.

\subsection{Quality Appraisal}

The quality of the economic evidence reported in included full publications was assessed using the 36-item checklist of the National Institute for Health and Care Excellence (NICE) Single Technology Appraisal Specification for submission of evidence (January 2015), adapted from Drummond and Jefferson [35]. This tool was used to assess the EE design, data collection methods, and analysis and interpretation of the results. The quality of conference abstracts and posters was not evaluated as they generally contain insufficient information to allow quality to be assessed rigorously. 


\section{Results}

\subsection{Identification of Included Economic Evaluations}

The number of citations retrieved and subsequently included in the analysis is summarised in Supplementary Fig. 1 (see the ESM). A total of 217 citations were retrieved from the electronic database search; 178 were subsequently excluded, and full publications were obtained for the remaining 39 citations. Based on review of the full publications, an additional 13 citations were excluded (Supplementary Table 4). Thus, 26 publications from the electronic database searches were included in the systematic literature review. Searches of conference proceedings and reference lists of the included publications identified a further nine citations that met the eligibility criteria. Thus, a total of 35 publications [26-28, 36-66], reporting data from 27 unique EEs, were included in the systematic literature review (Table 1).

Of the 13 EEs reported as full publications, six $[26,27,46,58,62,65]$ had previously been presented at a conference $[38,41,45,52,63,66]$ and posters were sourced for all except one [45]. For the remaining EEs, the data were reported in abstract $(n=7)[36,39,49,53,54,56,57,59]$ or poster form $(n=7)$ only [40, 43, 44, 47, 51, 60, 61, 64].

In the remainder of this article, EEs are referenced using the full manuscript, if one is available, otherwise the abstract or poster is referenced. One UK EE has two associated abstracts [53, 57]; in this case, the Planche abstract [57] is cited, as the key data were taken from this abstract. Also, one French EE has two associated posters [60, 61]; in this case, the 2014 poster is considered the key reference [60]. A summary of duplicate publications is provided in Supplementary Fig. 2 (see the ESM).

\subsection{Details of Included Economic Evaluations}

\subsubsection{Treatments Investigated}

Vancomycin was assessed in all 27 EEs, whereas fidaxomicin and metronidazole were investigated in 24 EEs [26-28, 36, 37, 39, 40, 42, 43, 46-51, 54, 56-60, 62, 64] and 13 EEs [37, 44, 46-49, 51, 54, 55, 57, 59, 62, 65], respectively. In two EEs [37, 57], vancomycin and metronidazole were combined in a single treatment arm and the intervention used was based on CDI severity or $C$. difficile strain. FMT was not a comparator of interest, but was a treatment arm in six of the EEs included [46, 48, 51, 54, 59, 65].

\subsubsection{Treatment Comparisons}

The most common treatment comparison was between fidaxomicin and vancomycin only, reported in 14 EEs
[26-28, 36, 39, 40, 42, 43, 50, 56, 58, 60, 64]. Five EEs [37, 47, 49, 57, 62] compared fidaxomicin with vancomycin and metronidazole. FMT was compared to the three antibiotics in five EEs [46, 48, 51, 54, 59] and to vancomycin and metronidazole alone in one EE [65]. Vancomycin and metronidazole only were compared in two EEs [44, 55].

\subsubsection{Model Types}

A Markov model was used in ten EEs [26, 27, 47, 48, 50, $56,58,60,62,64]$, and a decision-tree model was used in 11 EEs [28, 36, 37, 40, 43, 46, 51, 54, 55, 65]. Four EEs $[39,44,49,59]$ used a decision-analytic model, but no further details were provided in the publications. The two remaining EEs were not model-based, but used patient data from the authors' institutions [42, 57].

Five of the EEs comparing fidaxomicin and vancomycin $[26,47,58,62,64]$ were based on country adaptations of the same model (see the ESM, Supplementary Fig. 2).

\subsubsection{Economic Evaluation Perspectives}

Twelve countries were represented across the 27 EEs (see the ESM, Supplementary Fig. 3). These included the USA (11 EEs [28, 36, 37, 42, 44, 46, 49, 51, 54, 59, 65]), Canada (three EEs $[48,55]$ ), the UK (two EEs [26, 57]) and Germany (two EEs [27, 43]). Overall, 24 of the EEs were conducted from a payer perspective [26-28, 36, 37, 39, 40, $42,44,47-50,54-60,62,64,65]$ and three $[43,46,51]$ were conducted from a societal perspective.

\subsubsection{Willingness-to-Pay Thresholds}

WTP thresholds for the base-case analysis were reported in nine EEs [26-28, 37, 46, 48, 50, 54, 60]. The WTP thresholds were US $\$ 100,000 /$ QALY [28], US $\$ 50,000 /$ QALY [37, 46] and US\$5,000/QALY [54] in analyses conducted from the USA perspective. In other EEs, WTP thresholds were CAN\$50,000/QALY (Canada) [48], $€ 50,000 / \mathrm{QALY}$ (France [60] and Germany [27]), and $£ 20,000$ and $£ 30,000$ (Scotland) [26]. In the final EE, the WTP threshold was 53,307,040.00 Republic of Serbia dinars per life-year saved [50].

\subsubsection{Sponsorship of Economic Evaluations}

Information regarding sponsorship was available for 19 analyses. Nine [26, 27, 40, 47, 57, 58, 60, 62, 64] were sponsored by the marketing authorisation holder of fidaxomicin [Merck (previously Optimer Pharmaceuticals then Cubist Pharmaceuticals) in the USA; Astellas in Europe 


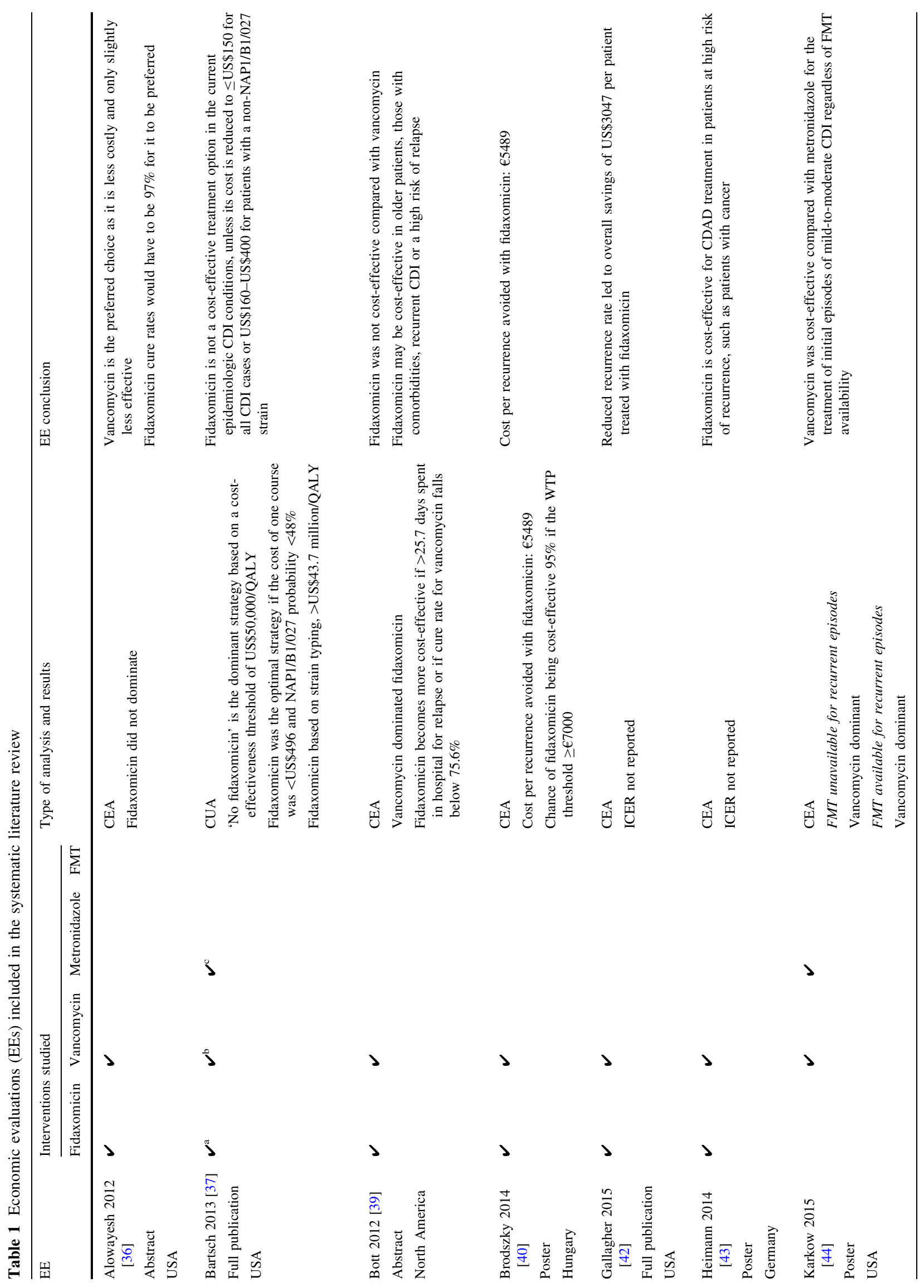




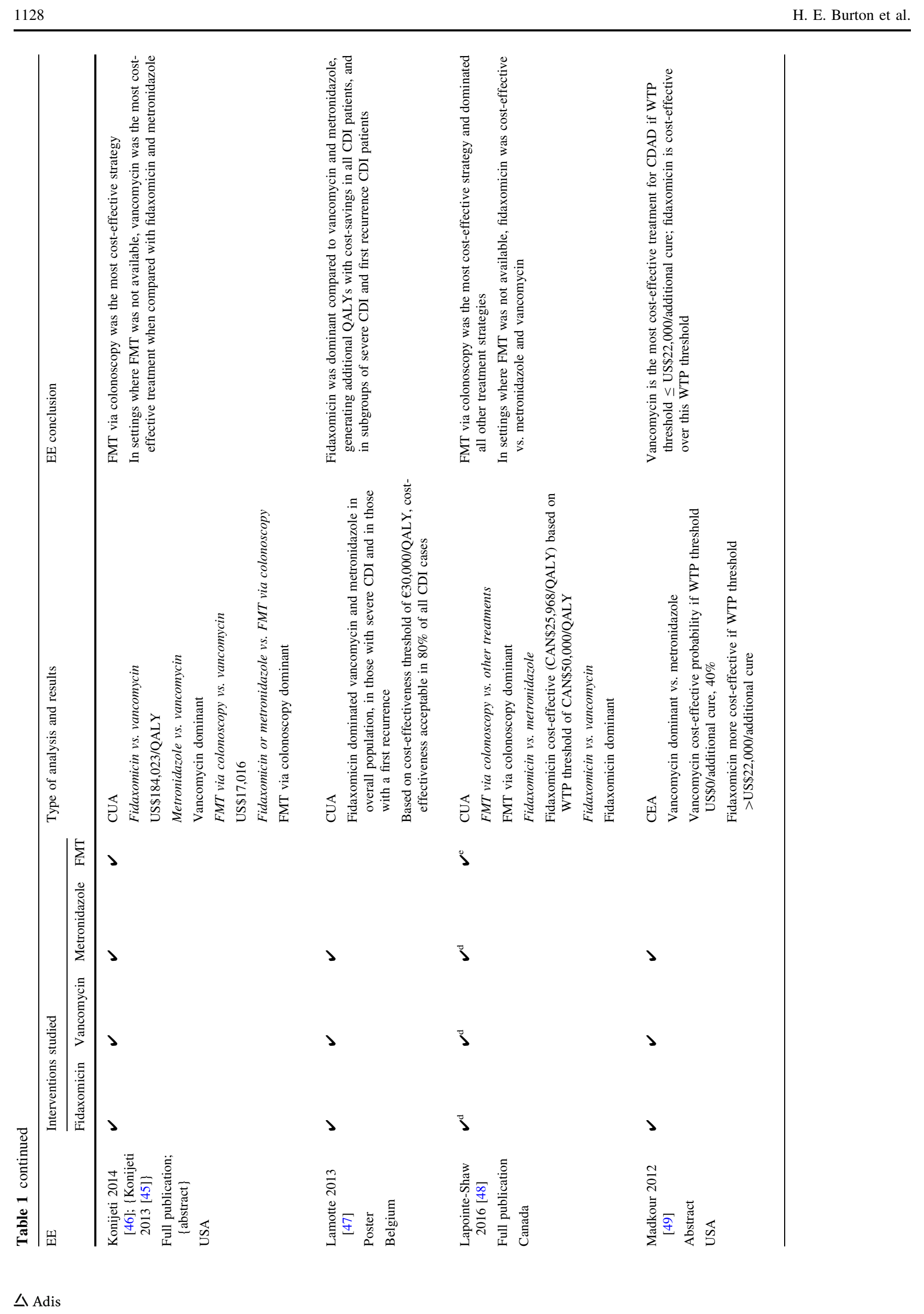




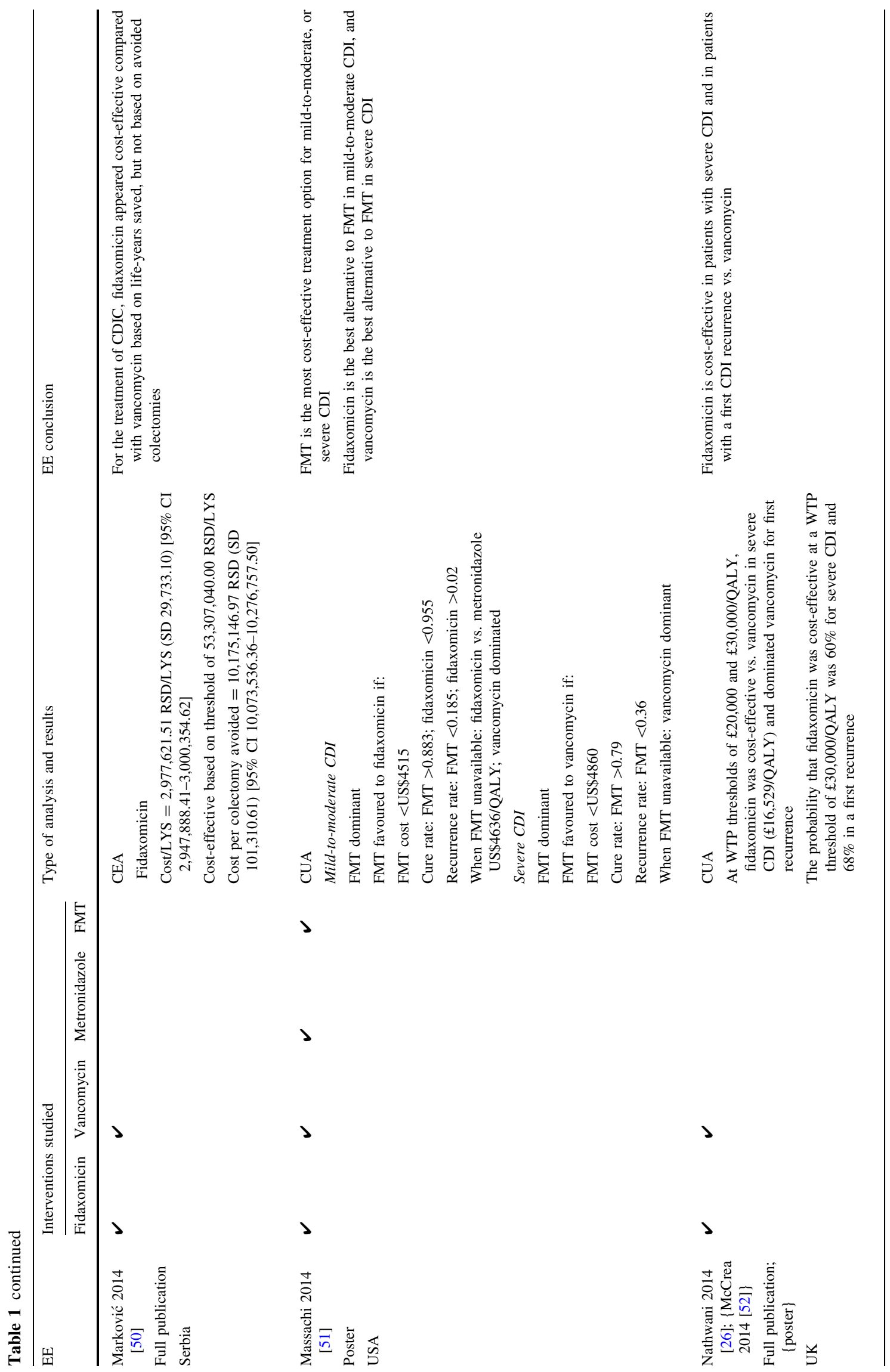




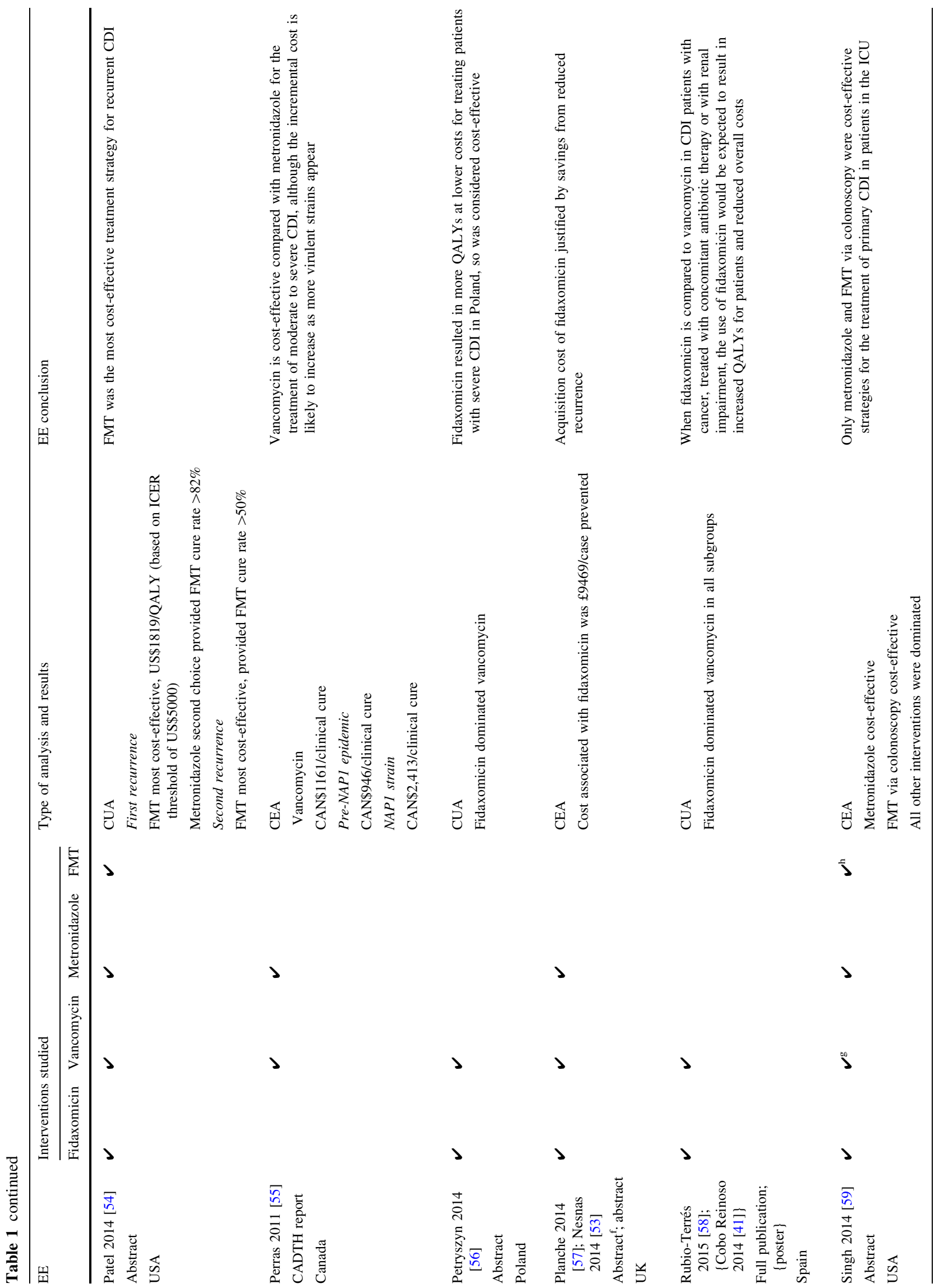




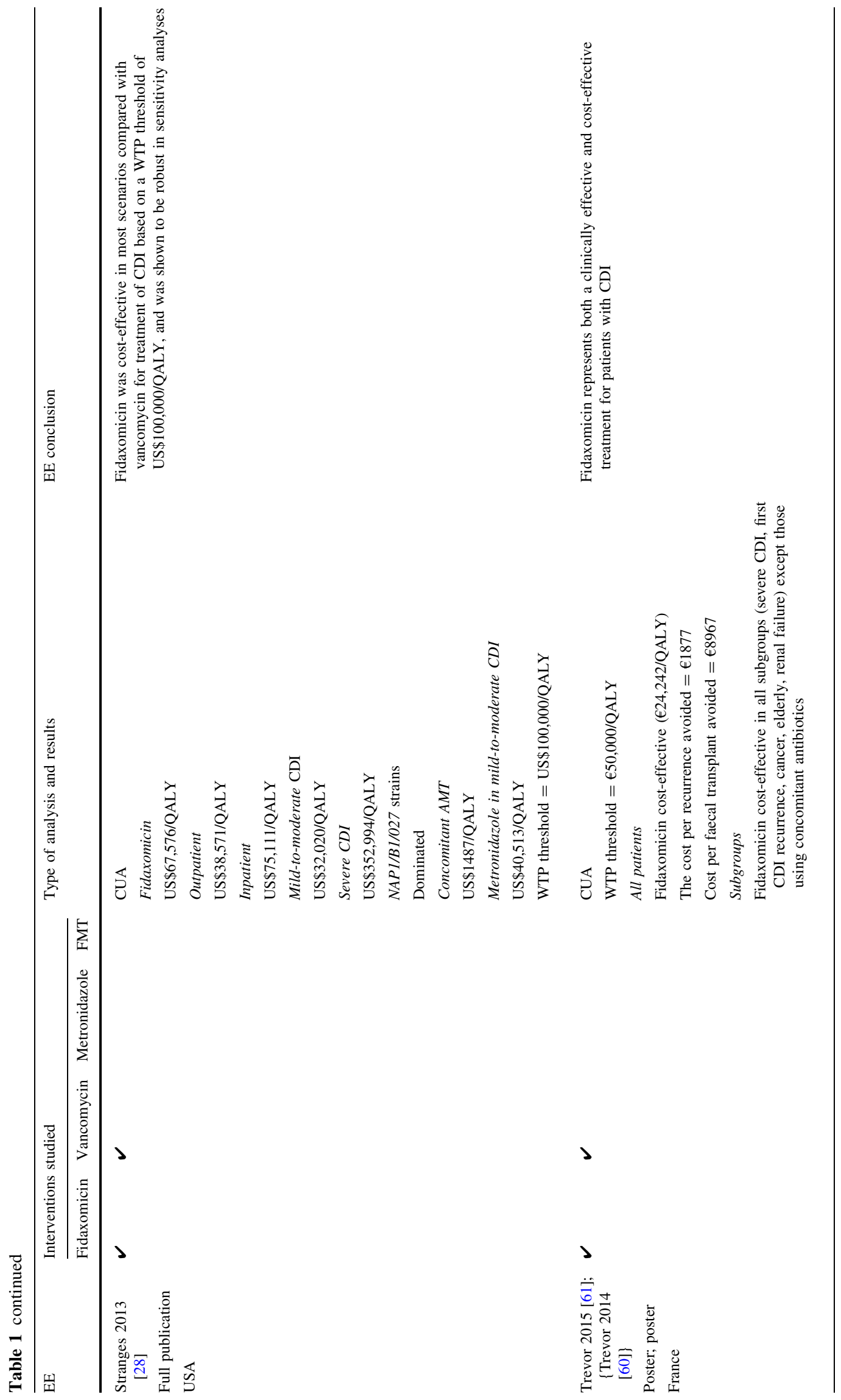




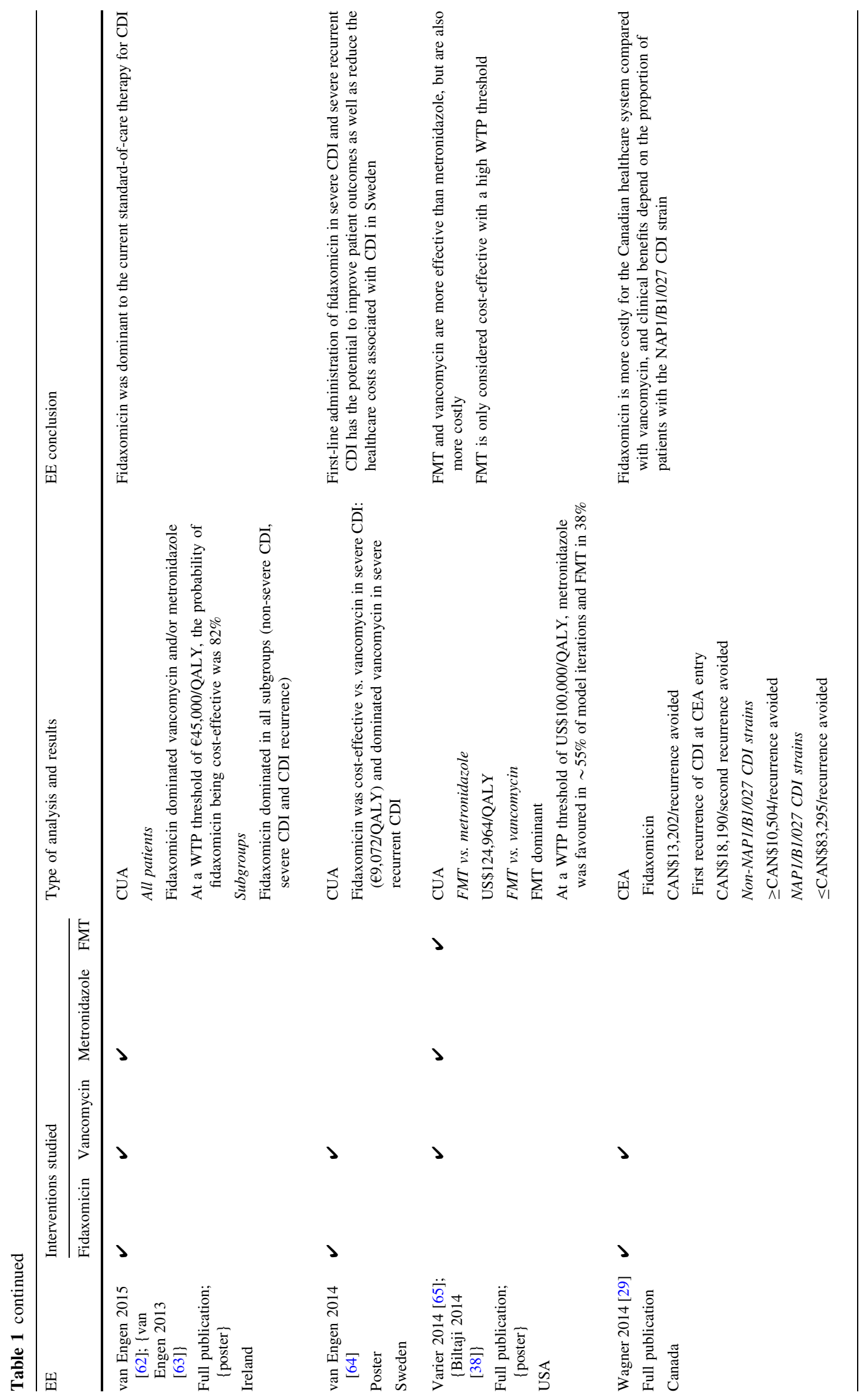




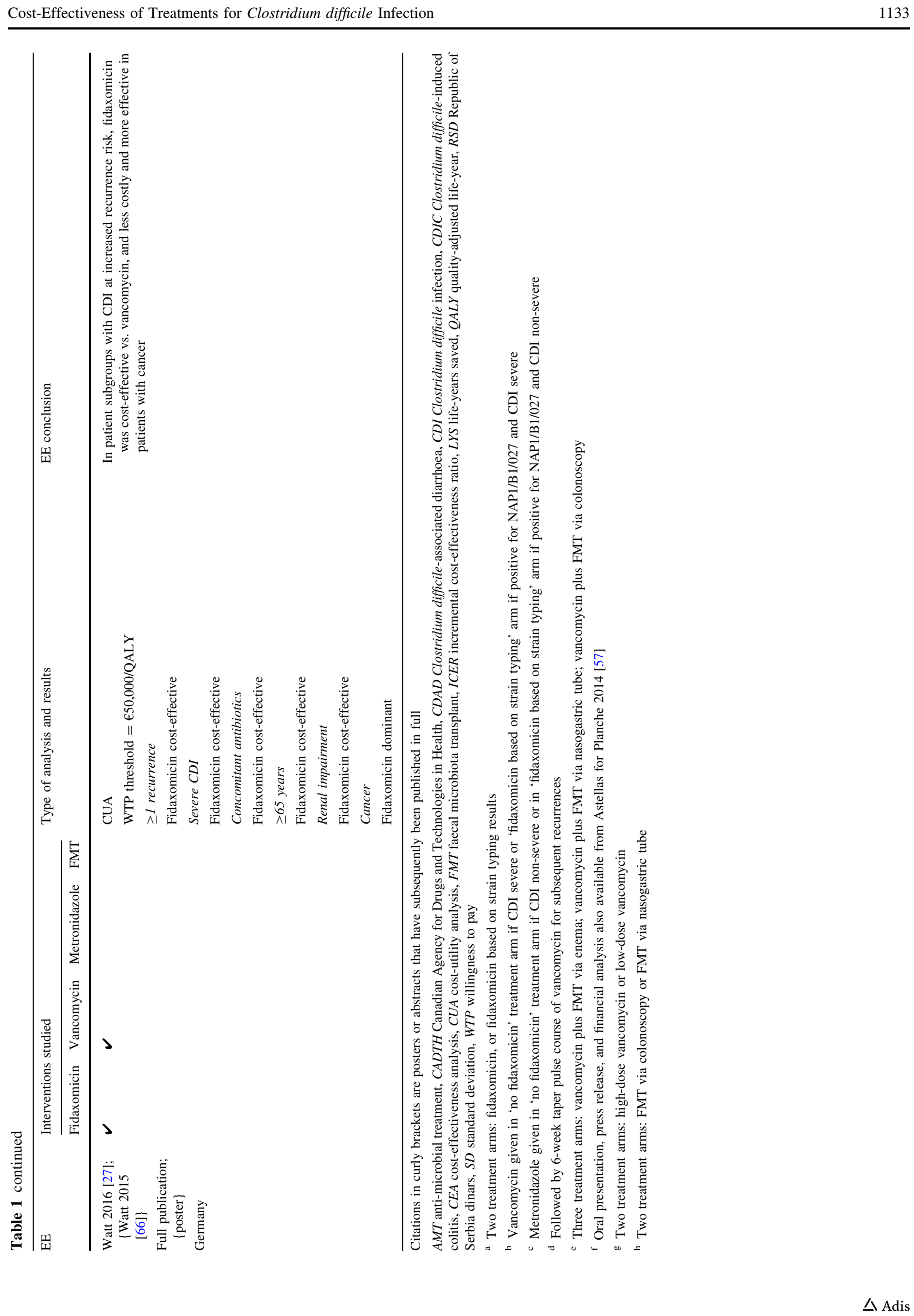


and Japan]. Of the remaining analyses, funding (or partfunding) by industry was confirmed for only two [43].

\subsection{Quality Appraisal Results}

Quality appraisal of all 13 full publications was conducted [26-28, 37, 42, 46, 48, 50, 55, 58, 62, 65]. In general, EEs were of good quality and reported in sufficient detail (see the ESM, Supplementary Table 5). EE design and analysis of results were generally well described, but reporting of data collection methodology was inconsistent between EEs. In particular, details of sources for effectiveness estimates, quantities of resource use, and justifications for the choice of model were only reported in a minority of publications.

\subsection{Results of Economic Evaluations}

The results and conclusions of each of the 27 EEs are summarised in Table 1.

\subsubsection{Overall CDI Population}

Figure 1 shows the results of the 27 EEs included. Fidaxomicin was cost-effective versus vancomycin or metronidazole in 14 of the 24 EEs (58.3\%) in which it was evaluated [26-28, 40, 42, 43, 47, 50, 56-58, 60, 62, 64]. Five of the 24 EEs included FMT; when data for this therapy were excluded, fidaxomicin was found to be costeffective in one of the EEs [48]. When this scenario was added to the other fidaxomicin EEs, 15 of 24 EEs (62.5\%) found fidaxomicin to be cost-effective versus vancomycin or metronidazole [26-28, 40, 42, 43, 47, 48, 50, 56-58, 60, $62,64]$. When the analysis was restricted to data published in full manuscripts only (i.e. when data from abstracts/posters were excluded), fidaxomicin was cost-effective versus vancomycin or metronidazole in seven of 11 (63.6\%) EEs [26-28, 42, 50, 58, 62] and cost-effectiveness was unclear in one study [29]. When FMT data were excluded, fidaxomicin was cost-effective in one additional study [48], i.e. in eight of 11 (72.7\%) EEs overall.

Vancomycin was cost-effective versus fidaxomicin or metronidazole in five of the 27 EEs (18.5\%) $[36,39,44,49,55]$. Six of the 27 EEs included FMT, and when data for this therapy were excluded, vancomycin was found to be cost-effective in one of the EEs [46]. When this scenario was added to the other vancomycin EEs, six of 27 EEs $(22.2 \%)$ found vancomycin to be cost-effective versus fidaxomicin or metronidazole [36, 39, 44, 46, 49, 55]. When the analysis was restricted to data published in full manuscripts only, vancomycin was cost-effective versus fidaxomicin or metronidazole in one of 13 (7.7\%) EEs [55]. When FMT data were excluded, vancomycin was cost-

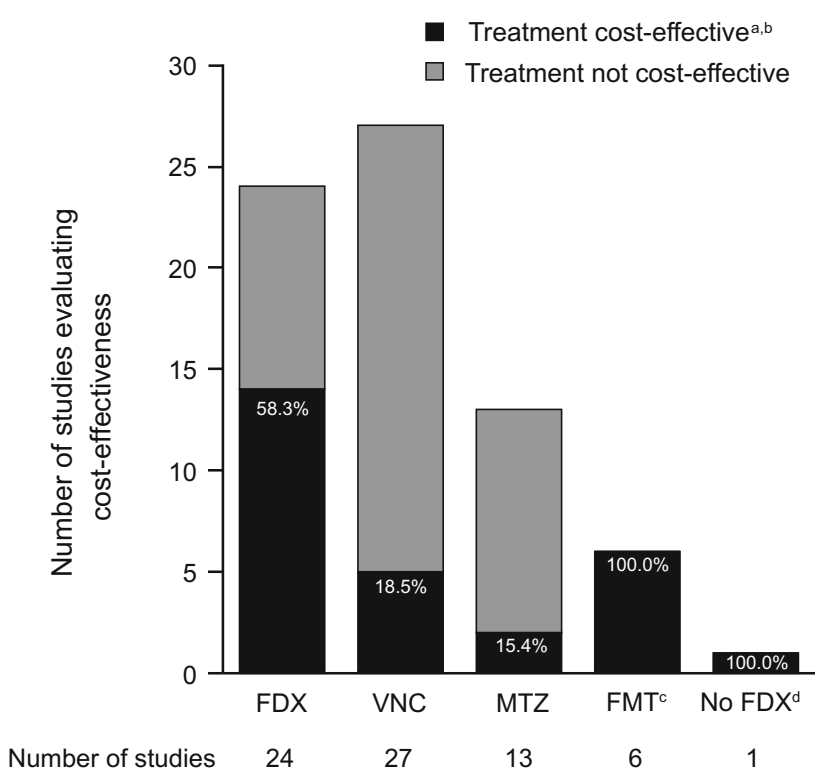

Fig. 1 Number and percentage of EEs reporting positive and negative cost-effectiveness results for each treatment for CDI. $C D I$ Clostridium difficile infection, EE economic evaluation, FDX fidaxomicin, FMT faecal microbiota transplant, MTZ metronidazole, VNC vancomycin. ${ }^{\mathrm{a}}$ Number of EEs is $>27$, as two EEs reported $>1$ treatment to be cost-effective [65]. ${ }^{\mathrm{b}}$ Note that cost-effectiveness thresholds vary according to EE and region. ${ }^{c}$ FMT was evaluated in six EEs only, but there were multiple comparisons with other treatments in some EEs (also note that FMT was not included as a search term in the systematic literature review). ${ }^{\mathrm{d}} \mathrm{EE}$ compared FDX with 'no FDX' strategy (VNC or MTZ) [37]. One EE was not included in the figure as the cost-effectiveness conclusion was unclear from the publication [29]

effective in one additional study [46], i.e. in two of 13 $(15.4 \%)$ EEs overall.

Metronidazole was cost-effective versus fidaxomicin, vancomycin or FMT in two of the 13 EEs $(15.4 \%)[59,65]$. When data for FMT were excluded, metronidazole was found to be cost-effective in one further EE [54]. When this scenario was added to the other metronidazole EEs, three of 13 EEs (23.1\%) found metronidazole to be cost-effective versus fidaxomicin, vancomycin or FMT [54, 59, 65]. When the analysis was restricted to data published in full manuscripts only, metronidazole was cost-effective in one of five (20.0\%) EEs [65].

The 'vancomycin or metronidazole' strategy (treatment dependent on CDI severity or $C$. difficile strain) was costeffective versus fidaxomicin in the one EE in which it was evaluated [37]. FMT was cost-effective versus at least two of the three antibiotics (fidaxomicin, vancomycin, metronidazole) in all six EEs in which it was evaluated [46, 48, 51, 54, 59, 65].

The cost-effectiveness of interventions according to specific treatment comparisons, as reported in the included 


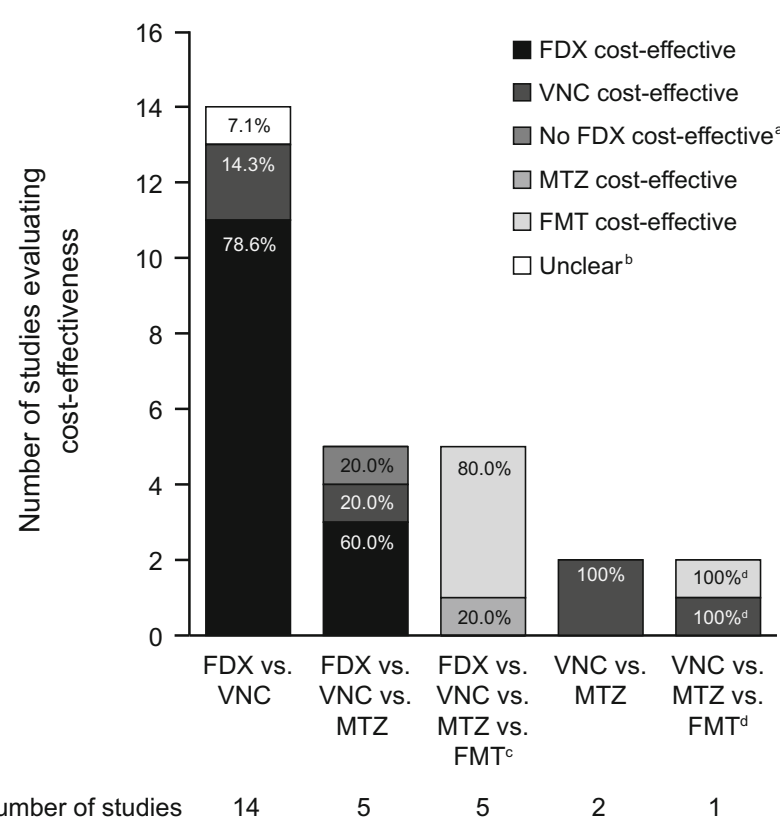

Fig. 2 Number of EEs reporting positive cost-effectiveness results for each treatment for CDI, according to specific treatment comparisons. Numbers below x-axis refer to the number of EEs reporting each treatment comparison. CDI Clostridium difficile infection, EE economic evaluation, $F D X$ fidaxomicin, $F M T$ faecal microbiota transplant, $M T Z$ metronidazole, $V N C$ vancomycin. ${ }^{a} \mathrm{EE}$ compared FDX with 'no FDX' strategy (VNC or MTZ) [37]. ' Cost-effectiveness conclusion unclear from publication [29]. ${ }^{\mathrm{c} B o t h}$ FMT and MTZ were considered to be cost-effective [59]. ${ }^{\mathrm{d}} \mathrm{MTZ}$ cost-effective vs. FMT, and FMT cost-effective vs. VNC [65]

EEs, is summarised in Fig. 2. In the 14 EEs that compared only fidaxomicin and vancomycin, fidaxomicin was costeffective in $11(78.6 \%)$ [26-28, 40, 42, 43, 50, 56, 58, 60, 64]. In the five EEs comparing fidaxomicin, vancomycin and metronidazole, fidaxomicin was cost-effective in three $(60 \%)[47,62]$.

The reported key cost-effectiveness drivers were rate of recurrence $[26-28,40,47,51,60,62,64]$, drug costs $[37,46,51,59,65]$, cure rate $[27,28,36,39,46,51,54,59$, 64, 65], time horizon [47] and length of hospitalisation and associated costs $[26,28,36,39,55,62,64]$. Cure rates related to clinical cure in three of the EEs [27, 36, 64]; in the remaining EEs, it was unclear whether cure rates related to clinical cure or sustained cure.

\subsubsection{Patient Subgroups}

Sixteen of the EEs [26-28, 39, 43, 46-48, 51, 54, 56, 58, $60,62,64]$ included in the systematic literature review evaluated the cost-effectiveness of CDI interventions in the patient subgroups of interest (recurrent CDI; those at higher risk of mortality or CDI recurrence; severe CDI). In 12 of these $[26,27,39,43,46,48,51,54,56,58,62]$, the analysis focused on the subgroup(s) of interest, while in the remaining four $[28,47,60,62]$, the overall CDI population was used for the base case analysis, with additional analyses in the subgroups of interest. Fidaxomicin was costeffective in most of the EEs reporting subgroup results (Fig. 3).

\section{Discussion}

This systematic literature review identified 27 unique analyses that evaluated the cost-effectiveness of antibiotics used to treat CDI. In most of the EEs included, fidaxomicin was cost-effective compared with vancomycin or metronidazole. Similar results were obtained when the analyses were conducted in subgroups of patients with recurrent or severe $\mathrm{CDI}$, and in those at higher risk of recurrence or mortality. Although this review has some limitations (which are discussed below), the results of the EEs are consistent with the clinical profile of fidaxomicin and the current evidence describing the costs of managing CDI. Two phase III, randomised, double-blind trials [21,22] show a significant improvement in recurrence rates and sustained cure rates in favour of fidaxomicin versus vancomycin. While there are no direct comparative trials versus metronidazole, the results of an indirect treatment comparison indicate that fidaxomicin reduced recurrence and increased sustained cure versus metronidazole [23]. These clinical data are consistent with the pharmacological profile of fidaxomicin, which has minimal effects on the gut flora and has a greater preventative effect on $C$. difficile sporulation and subsequent cell growth from spores, compared with the other two antibiotics $[16,67]$. Given the significant economic impact associated with prolonged hospitalisation in patients with CDI, particularly for recurrent episodes [13-15], the results of the current systematic literature review indicate that fidaxomicin provides clinical and economic benefits despite having a higher acquisition cost.

FMT was cost-effective versus antibiotic treatment in all six of the EEs that were included. However, this procedure is only listed by guidelines for multiple recurrences $[32,33]$ and is conducted by only a minority of hospitals in Europe. Consequently, it was not included as a comparator of interest in the current analysis, so not all studies evaluating the cost-effectiveness of FMT will have been identified. In addition, information relating to specific methodology for FMT and the cost of carrying out the procedure is not publicly available and will vary from centre to centre. For this reason, it is difficult to evaluate the cost-effectiveness of FMT.

Speculating on the reasons why some EEs showed a particular CDI treatment to be cost-effective while others did not is difficult in view of the heterogeneity of the model 
(a) Patients with recurrent $\mathrm{CDI}$

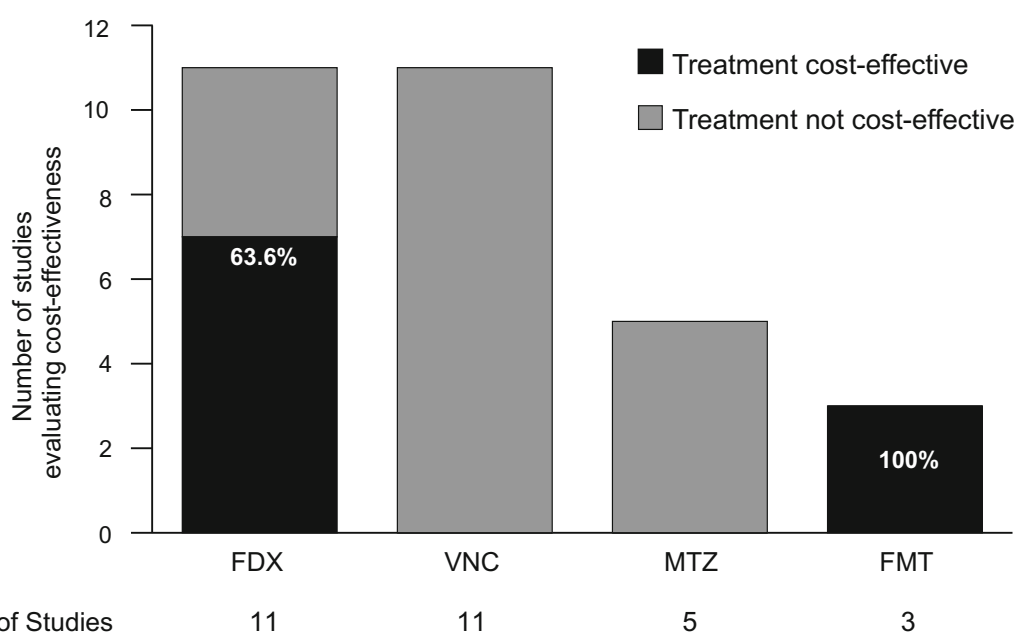

(b) Patients at higher risk of mortality or CDI recurrence

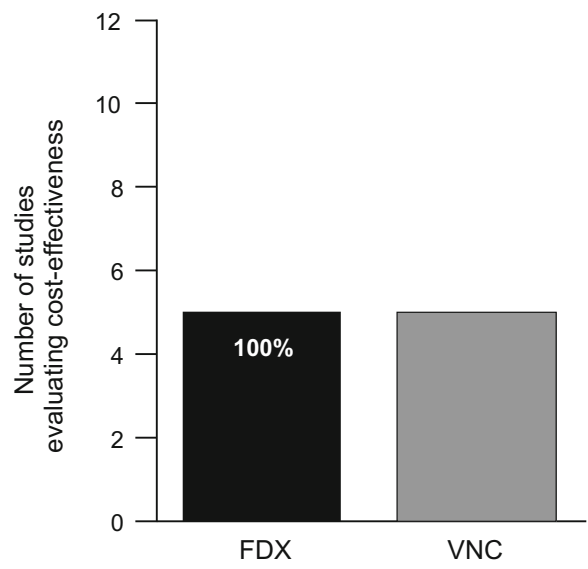

Number of Studies

5

5

(c) Patients with severe CDI

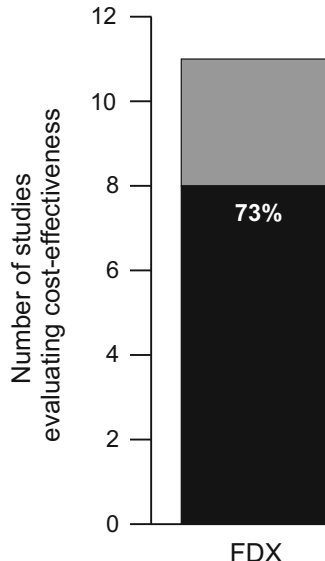

11

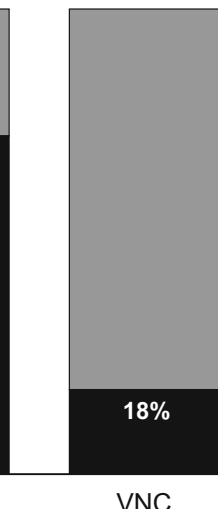

11

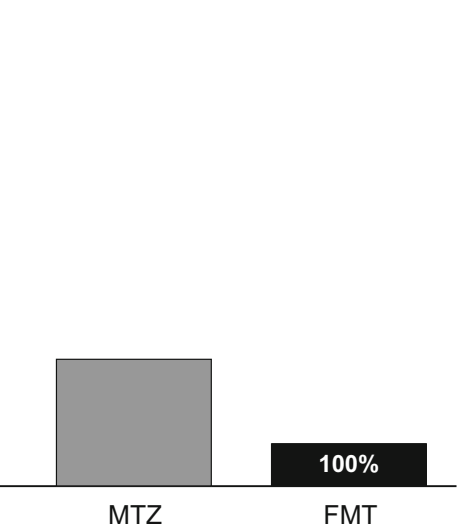

1

Fig. 3 Number and percentage of EEs reporting positive and negative cost-effectiveness results for each treatment in patient subgroups of interest. CDI Clostridium difficile infection, $F D X$ fidaxomicin, $F M T$ faecal microbiota transplant, $M T Z$ metronidazole, $V N C$ vancomycin 
input parameters, as well as the lack of information on these for some of the analyses published in abstract form. However, it may reflect differences in model input parameters for rates of recurrence, drug costs, cure rate, time horizon and length and cost of hospitalisation, as these were identified as key cost-effectiveness drivers in the EEs included. The issue of variability in the results of EEs has been highlighted previously, and one of the most commonly cited factors for generating variability in results between locations is the unit costs associated with particular resources [68].

Results of a recently published cost-of-illness study conducted in a German hospital indicate that the costs of recurrence may have been underestimated previously [69]. In that particular study, the direct treatment costs of CDI recurrence were estimated at $€ 73,898$ per patient with one or more recurrence [69]; this is four times greater than the incremental cost estimates for recurrent CDI reported in the US-based systematic review mentioned above [13]. It has also been shown that recurrent CDI has a greater impact on patients' quality of life than the initial episode [70]. Taken together, these data suggest that fidaxomicin may be more cost-effective than suggested by the results of our systematic literature review of EEs. However, translating these cost-effectiveness results into different payer systems can be challenging. First, the incidence (and therefore economic impact) of recurrence may be underestimated, reflecting, in part, differences in the definition of recurrence between hospitals [71]. Also, in certain settings, patients may present at different centres for initial and recurrent episodes [69]; as budgetary control is often held at the hospital rather than regional level, the payer for the initial episode will not benefit from the reduction in later costs associated with reduced recurrence. Even if a patient is treated at the same hospital, there may be no financial penalty for recurrence, and consequently, individual hospitals may be less likely to use treatments with higher acquisition costs, but lower recurrence rates.

European guidelines for the management of CDI make treatment recommendations based on the severity of the infection and whether the infection is recurrent [32]. For non-severe cases, metronidazole is recommended over fidaxomicin and vancomycin, while for recurrent cases, equal weight is given to fidaxomicin and vancomycin over metronidazole [32]. In the USA, guidelines recommend metronidazole for mild-to-moderate CDI and vancomycin for severe infection [33]. Although the World Society of Emergency Surgery recognises the place of fidaxomicin in patients with mild-to-moderate CDI at high risk of recurrence, it currently recommends fidaxomicin and vancomycin with equal weight for those with multiple recurrences [72]. These recommendations are based on clinical data only and do not take cost-effectiveness into account. The current systematic literature review is informative in this regard, particularly in view of increased pressures to reduce costs in the healthcare sector. In Australia, treatment recommendations for CDI do take costeffectiveness into consideration and fidaxomicin is recommended for those with multiple recurrences if they have a high risk of subsequent recurrence [72]. Conversely, the Australian guidelines recommend that fidaxomicin is reserved for third-line use in severe, first-episode CDI due to uncertainty about cost-effectiveness versus conventional treatment options [72]. In the study by Watt et al., which is included in the current analysis, fidaxomicin was shown to be cost-effective versus vancomycin and metronidazole in those with an initial CDI episode and in those with severe CDI, albeit from the perspective of the NHS in England [25].

The analysis does have some limitations. These include limitations associated with the models used in the EEs (e.g. extrapolation of the outcomes to the model timelines) as well as limitations associated with the overall analysis, such as its non-quantitative nature and the heterogeneity of the EEs included, which varied according to the type of model used, the interventions analysed, the time horizons employed, the outcome measures reported, and the country perspectives from which the EEs were conducted. Country preferences vary in terms of drug acquisition costs, hospitalisation costs and how cost-effectiveness is assessed (e.g. cost/QALY, cost/recurrence avoided, cost/clinical cure); WTP thresholds, which generally equate to one to three times a country's gross domestic product [73], also vary, with wealthier countries having a higher threshold. Thus, in countries with lower cost-effectiveness thresholds, fidaxomicin may not be interpreted to be cost-effective despite its beneficial clinical effects on recurrence and sustained cure. It should also be noted that in the USA, WTP thresholds are not formally used to make treatment decisions. Another limitation of the current analysis is that five of the EEs comparing fidaxomicin and vancomycin [26, 47, 58, 62, 64] were based on country adaptations of the same model and therefore had similar inputs and assumptions. Also, some of the EEs were published in abstract or poster form only $[36,39,40,43,44,47,49,51,53,54,56,57,59-61,64]$ and therefore contain only limited information on the details of the economic models; however, excluding these publications could potentially have introduced bias to the results and would have contravened the methodological guidelines of the Cochrane Collaboration [31]. Nevertheless, when the analysis was restricted to data published in full manuscripts only, similar results were obtained to those for the full analysis. Finally, the 'grey literature' (e.g. data in company repositories, regulatory agency digital archives, industry investor reports and press releases, and institutional websites) was not searched, and therefore some relevant 
assessments of cost-effectiveness in CDI may have been omitted (i.e. publication bias may have been introduced). On the other hand, a strength of the analysis is that selection bias was avoided by a priori documentation of criteria for EE selection. Also, despite its limitations, this systematic literature review is the first to summarise the currently available information on the cost-effectiveness of antibiotic treatment for CDI. As such, it provides a general overview that may be useful for aiding treatment decisions.

In conclusion, the results of this systematic literature review indicate that in most EEs, fidaxomicin was costeffective versus metronidazole or vancomycin in patients with CDI. Fidaxomicin was also cost-effective in most of the EEs that evaluated specific, higher-risk patient subgroups. In order to build on these data, updated indirect treatment comparison network analyses of the clinical effectiveness of antibiotic treatments for CDI would be desirable to allow a more integrated EE. Nevertheless, results from the current analysis are highly relevant given the high budgetary impact of managing CDI and the increasing restrictions on healthcare budgets. However, in order for the cost offsets of fidaxomicin to be realised, the true extent and impact of recurrent CDI must be more fully understood. Also, there needs to be a shift in focus from cost containment at the individual hospital level to cost evaluation at a national level. If these barriers can be addressed, first-line use of fidaxomicin has the potential to facilitate more efficient use of funds in clinical practice.

Acknowledgements We thank Nicky French, $\mathrm{PhD}$ (on behalf of Bioscript Medical) for medical writing support, which was funded by Astellas Pharma Inc. The data in this manuscript were presented, in part, at the ISPOR 21st Annual International Meeting Washington, D.C., USA (21-25 May 2016) [Burton HE, Mitchell SA, Watt M. Value Health 2016;19(3):A218].

Author Contributions Analysis concept and design: HB, SM, MW; data acquisition: HB, SM; analysis and interpretation of data: HB, SM, MW; drafting, review and approval of publication: HB, SM, MW; accountability for all aspects of the work: HB, SM, MW.

\section{Compliance with Ethical Standards}

Data availability statement All relevant data are included in the manuscript or supplementary material.

Funding These analyses were initiated and funded by Astellas Pharma Inc.

Conflict of interest HB and SM are employees of DRG Abacus, which received payment from Astellas Pharma Inc. for conducting the analysis. MW was an employee of Astellas Pharma Inc at the time of manuscript preparation.

Open Access This article is distributed under the terms of the Creative Commons Attribution-NonCommercial 4.0 International License (http://creativecommons.org/licenses/by-nc/4.0/), which permits any noncommercial use, distribution, and reproduction in any medium, provided you give appropriate credit to the original author(s) and the source, provide a link to the Creative Commons license, and indicate if changes were made.

\section{References}

1. Le Monnier A, Zahar JR, Barbut F. Update on Clostridium difficile infections. Medecine et maladies infectieuses. 2014;44(8):354-65.

2. Rodriguez C, Van Broeck J, Taminiau B, Delmee M, Daube G. Clostridium difficile infection: early history, diagnosis and molecular strain typing methods. Microb Pathog. 2016;97:59-78.

3. Shields K, Araujo-Castillo RV, Theethira TG, Alonso CD, Kelly CP. Recurrent Clostridium difficile infection: from colonization to cure. Anaerobe. 2015;34:59-73.

4. Kwon JH, Olsen MA, Dubberke ER. The morbidity, mortality, and costs associated with Clostridium difficile infection. Infect Dis Clin N Am. 2015;29(1):123-34.

5. Wiegand PN, Nathwani D, Wilcox MH, Stephens J, Shelbaya A, Haider S. Clinical and economic burden of Clostridium difficile infection in Europe: a systematic review of healthcare-facilityacquired infection. J Hosp Infect. 2012;81(1):1-14.

6. Kelly CP. Can we identify patients at high risk of recurrent Clostridium difficile infection? Clin Microbiol Infect. 2012;18(Suppl 6):21-7.

7. Ofosu A. Clostridium difficile infection: a review of current and emerging therapies. Ann Gastroenterol Q Publ Hell Soc Gastroenterol. 2016;29(2):147-54.

8. Vardakas KZ, Polyzos KA, Patouni K, Rafailidis PI, Samonis G, Falagas ME. Treatment failure and recurrence of Clostridium difficile infection following treatment with vancomycin or metronidazole: a systematic review of the evidence. Int $\mathbf{J}$ Antimicrob Agents. 2012;40(1):1-8.

9. Vincent Y, Manji A, Gregory-Miller K, Lee C. A review of management of Clostridium difficile infection: primary and recurrence. Antibiotics (Basel, Switzerland). 2015;4(4):411-23.

10. Leong C, Zelenitsky S. Treatment strategies for recurrent Clostridium difficile infection. Can J Hosp Pharm. 2013;66(6):361-8.

11. van Nood E, Speelman P, Kuijper EJ, Keller JJ. Struggling with recurrent Clostridium difficile infections: is donor faeces the solution? Euro surveillance: bulletin Europeen sur les maladies transmissibles (European communicable disease bulletin). 2009;14(34). pii: 19316.

12. Fitzpatrick F, Barbut F. Breaking the cycle of recurrent Clostridium difficile infections. Clin Microbiol Infect. 2012;18(Suppl 6):2-4.

13. Ghantoji SS, Sail K, Lairson DR, DuPont HL, Garey KW. Economic healthcare costs of Clostridium difficile infection: a systematic review. J Hosp Infect. 2010;74(4):309-18.

14. Al-Eidan FA, McElnay JC, Scott MG, Kearney MP. Clostridium difficile-associated diarrhoea in hospitalised patients. J Clin Pharm Ther. 2000;25(2):101-9.

15. Bouza E. Consequences of Clostridium difficile infection: understanding the healthcare burden. Clin Microbiol Infect. 2012;18(Suppl 6):5-12.

16. Babakhani F, Bouillaut L, Gomez A, Sears P, Nguyen L, Sonenshein AL. Fidaxomicin inhibits spore production in Clostridium difficile. Clin Infect Dis. 2012;55(Suppl 2):S162-9.

17. Babakhani F, Gomez A, Robert N, Sears P. Killing kinetics of fidaxomicin and its major metabolite, OP-1118, against Clostridium difficile. J Med Microbiol. 2011;60(Pt 8):1213-7. 
18. Tannock GW, Munro K, Taylor C, Lawley B, Young W, Byrne B, et al. A new macrocyclic antibiotic, fidaxomicin (OPT-80), causes less alteration to the bowel microbiota of Clostridium difficile-infected patients than does vancomycin. Microbiology (Reading, England). 2010;156(Pt 11):3354-9.

19. Housman ST, Thabit AK, Kuti JL, Quintiliani R, Nicolau DP. Assessment of Clostridium difficile burden in patients over time with first episode infection following fidaxomicin or vancomycin. Infect Control Hosp Epidemiol. 2016;37(2):215-8.

20. Chilton $\mathrm{CH}$, Crowther GS, Ashwin H, Longshaw CM, Wilcox MH. Association of fidaxomicin with C. difficile spores: effects of persistence on subsequent spore recovery, outgrowth and toxin production. PLoS One. 2016;11(8):e0161200.

21. Cornely OA, Crook DW, Esposito R, Poirier A, Somero MS, Weiss K, et al. Fidaxomicin versus vancomycin for infection with Clostridium difficile in Europe, Canada, and the USA: a doubleblind, non-inferiority, randomised controlled trial. Lancet Infect Dis. 2012;12(4):281-9.

22. Louie TJ, Miller MA, Mullane KM, Weiss K, Lentnek A, Golan $\mathrm{Y}$, et al. Fidaxomicin versus vancomycin for Clostridium difficile infection. N Engl J Med. 2011;364(5):422-31.

23. Cornely OA, Nathwani D, Ivanescu C, Odufowora-Sita O, Retsa $\mathrm{P}$, Odeyemi IA. Clinical efficacy of fidaxomicin compared with vancomycin and metronidazole in Clostridium difficile infections: a meta-analysis and indirect treatment comparison. J Antimicrob Chemother. 2014;69(11):2892-900.

24. Mullane K. Fidaxomicin in Clostridium difficile infection: latest evidence and clinical guidance. Ther Adv Chronic Dis. 2014;5(2):69-84.

25. Watt M, McCrea C, Posnett J, Berling M, Odeyemi IA. Costeffectiveness of fidaxomicin for the treatment of Clostridium difficile infection in England. Abstract presented at Federation of Infection Societies Annual Conference, Birmingham, UK, 11-13 Nov 2013.

26. Nathwani D, Cornely OA, Van Engen AK, Odufowora-Sita O, Retsa P, Odeyemi IA. Cost-effectiveness analysis of fidaxomicin versus vancomycin in Clostridium difficile infection. J Antimicrob Chemother. 2014;69(11):2901-12.

27. Watt M, McCrea C, Johal S, Posnett J, Nazir J. A cost-effectiveness and budget impact analysis of first-line fidaxomicin for patients with Clostridium difficile infection (CDI) in Germany. Infection. 2016;44(5):599-606.

28. Stranges PM, Hutton DW, Collins CD. Cost-effectiveness analysis evaluating fidaxomicin versus oral vancomycin for the treatment of Clostridium difficile infection in the United States. Value Health. 2013;16(2):297-304.

29. Wagner M, Lavoie L, Goetghebeur M. Clinical and economic consequences of vancomycin and fidaxomicin for the treatment of Clostridium difficile infection in Canada. Can J Infect Dis Med Microbiol. 2014;25(2):87-94.

30. Centre for Reviews and Dissemination. Systematic Reviews: CRD's guidance for undertaking reviews in health care 2009. https://www. york.ac.uk/media/crd/Systematic_Reviews.pdf. Accessed Aug 2017.

31. Higgins JPT, Green S. Cochrane handbook for systematic reviews of interventions. 2011. Version 5.1.0: http://handbook. cochrane.org. Accessed Aug 2017.

32. Debast SB, Bauer MP, Kuijper EJ. European society of clinical microbiology and infectious diseases: update of the treatment guidance document for Clostridium difficile infection. Clin Microbiol Infect. 2014;20(Suppl 2):1-26.

33. Surawicz CM, Brandt LJ, Binion DG, Ananthakrishnan AN, Curry SR, Gilligan PH, et al. Guidelines for diagnosis, treatment, and prevention of Clostridium difficile infections. Am J Gastroenterol. 2013;108(4):478-98.
34. Cohen SH, Gerding DN, Johnson S, Kelly CP, Loo VG, McDonald LC, et al. Clinical practice guidelines for Clostridium difficile infection in adults: 2010 update by the society for healthcare epidemiology of America (SHEA) and the infectious diseases society of America (IDSA). Infect Control Hosp Epidemiol. 2010;31(5):431-55.

35. Drummond MF, Jefferson TO. Guidelines for authors and peer reviewers of economic submissions to the BMJ. The BMJ economic evaluation working party. BMJ. 1996;313(7052):275-83.

36. Alowayesh M, Holdford D, Harpe SE. PIN35 decision analysis model evaluating the cost-effectiveness of fidaxomicin and vancomycin in the treatment of Clostridium difficile infection (CDI) from a hospital perspective. Value Health. 2012;15(4):A243.

37. Bartsch SM, Umscheid CA, Fishman N, Lee BY. Is fidaxomicin worth the cost? An economic analysis. Clin Infect Dis. 2013;57(4):555-61.

38. Biltaji E, Varier R, Smith K, Roberts M, Lafleur J, Nelson R. Cost-effectiveness analysis of treatment strategies for initial Clostridium difficile infection. Value Health. 2014;17(3):A38.

39. Bott Q, Rice L, Oh J, Sinha V, Boyer N. Cost-effectiveness of fidaxomicin for the treatment of severe Clostridium difficile infection in hospitalized patients in North America. Pharmacotherapy. 2012;32(10):e292.

40. Brodszky V, Strbak B, Baji P, Pentek M, Gulacsi L. Cost-effectiveness of fidaxomicin therapy for Clostridium difficile infection in Hungary. Value Health. 2014;17(7):A675.

41. Cobo Reinoso J, Grau Cerrato S, Mensa Pueyo J, Salavert Lletí M, Toledo A, Anguita P, et al. Economic evaluation of fidaxomicin for the treatment of Clostridium difficile infections (CDI) also known as Clostridium difficile-associated diarrhoea (CDAD) in Spain. Value Health. 2014;17:A674.

42. Gallagher JC, Reilly JP, Navalkele B, Downham G, Haynes K, Trivedi M. Clinical and economic benefits of fidaxomicin compared to vancomycin for Clostridium difficile infection. Antimicrob Agents Chemother. 2015;59(11):7007-10.

43. Heimann SM, Cornely OA, Vehreschild MJGT, Vehreschild JJ, editors. Pharmacoeconomical decision-tree analysis of Clostridium difficile-associated diarrhea in patients with cancer treated with fidaxomicin or vancomycin. Abstract presented at ICAAC, Washington DC, USA, 5-9 Sept 2014.

44. Karkow D, Schroeder M, Ince D, Ernst E, editors. Cost-effectiveness analysis of vancomycin versus metronidazole for management of initial episodes of Clostridium difficile infection in hospitalized patients. Abstract presented at IDWeek, San Diego, USA, 7-11 Oct 2015.

45. Konijeti G, Sauk J, Shime M, Ananthakrishnan A. Cost-effectiveness of competing strategies for recurrent Clostridium difficile infection. Am J Gastroenterol. 2013;108:S473.

46. Konijeti GG, Sauk J, Shrime MG, Gupta M, Ananthakrishnan AN. Cost-effectiveness of competing strategies for management of recurrent Clostridium difficile infection: a decision analysis. Clin Infect Dis. 2014;58(11):1507-14.

47. Lamotte M, Vellopoulou K, Richir K, Smets J, Grabe K, Coppens $\mathrm{K}$. The cost-utility of fidaxomicin as compared to current standard treatment in the management of Clostridium difficile infections in Belgium. Value Health. 2013;16:A498.

48. Lapointe-Shaw L, Tran KL, Coyte PC, Hancock-Howard RL, Powis J, Poutanen SM, et al. Cost-effectiveness analysis of six strategies to treat recurrent Clostridium difficile infection. PLoS One. 2016;11(2):e0149521.

49. Madkour N, Bounthavong M, Hsu D. A comparison of the costeffectiveness of fidaxomicin, metronidazole, and vancomycin, in the treatment of Clostridium difficile-associated disease. Value Health. 2012;15(4):2.

50. Marković M, Kostic M, Ilickovic I, Jankovic S. Cost-effectiveness comparison of fidaxomicin and vancomycin for treatment of 
Clostridium difficile infection: a Markov model based on data from a South West Balkan Country in Socioeconomic Transition. Value Health Reg Issues. 2014;4:8.

51. Massachi S, Hay J. Cost-effectiveness of various Clostridium difficile infection (CDI) treatments in patients with recurrent infections. Value Health. 2014;17(3):2.

52. McCrea C, Perera S, Van Engen A, Watt M, Nazir J. Economic evaluation of fidaxomicin compared with vancomycin in the treatment of Clostridium difficile infection. Value Health. 2014;17:A270.

53. Nesnas J, Whitney L, Ahmad A, Planche T. Cost-effectiveness of fidaxomicin as first-line treatment for Clostridium difficile infection. Abstract presented at ECCMID, Barcelona, Spain, 10-13 May 2014.

54. Patel SS, Grinspan A, Colombel J-F, Atreja A. Cost effectiveness analysis of fecal microbiota transplant and antibiotic treatment for recurrent Clostridium difficile infection. Gastroenterology. 2014;1:2

55. Perras C, Tsakonas E, Ndegwa S, Conly J, Valiquette L, Farrah K. Vancomycin or Metronidazole for treatment of Clostridium difficile infection: clinical and economic analyses. 2011. https://www.cadth.ca/vancomycin-or-metronidazoletreatment-clostridium-difficile-infection-clinical-and-economicanalyses. Accessed Aug 2017.

56. Petryszyn P, Well A. Cost-utility analysis of fidaxomicin compared to vancomycin in the management of severe Clostridium difficile infection in Poland. Value Health. 2014;17(7):A368.

57. Planche T, Whitney L, Ahmad A, Nesnas J. Experience with fidaxomicin as first-line treatment for $C$. difficile infection (CDI): clinical outcomes and cost-effectiveness. Am J Gastroenterol. 2014;109:S678.

58. Rubio-Terrés C, Cobo Reinoso J, Grau Cerrato S, Mensa Pueyo J, Salavert Lleti M, Toledo A, et al. Economic assessment of fidaxomicin for the treatment of Clostridium difficile infection (CDI) in special populations (patients with cancer, concomitant antibiotic treatment or renal impairment) in Spain. Eur J Clin Microbiol Infect Dis. 2015;34(11):2213-23.

59. Singh P, Udeh B, Dalton J, Udeh C, Hata J. Cost-effectiveness of 6 treatments for primary Clostridium difficile infection in an ICU population. Crit Care Med. 2014;1:A1474.

60. Trevor N, Watt M, Tilleul P, Le Monnier A, Fau I, Ait-Yahia M, et al. Economic evaluation of fidaxomicin for the treatment of Clostridium difficile infections in France. Abstract presented at Healthcare Infection Society Conference, Lyon, France, 16-18 Nov 2014.

61. Trevor N, Watt M, Tilleul P, Le Monnier A, Fau I, Ait-Yahia M, et al. Cost-consequence analysis on the use of fidaxomicin and vancomycin to treat Clostridium difficile infection (CDI) in France. Abstract presented at ECCMID, Copenhagen, Denmark, 25-28 Apr 2015
62. Van Engen A, Casamayor M, Loftus F, Coen M, Garnham A, Watt $\mathrm{M}$, et al. Economic evaluation of fidaxomicin for the treatment of Clostridium difficile infection (C. difficile-associated diarrhoea) in Ireland. $\mathbf{J}$ Health Econ Outcomes Res. 2015;2:192-2026.

63. Van Engen A, Casamayor M, Loftus F, Crimin E, Lacey L. Economic evaluation of fidaxomicin for the treatment of Clostridium difficile infections (CDI) also known as $C$. difficileassociated diarrhoea (CDAD) in Ireland. Value Health. 2013; 16:A353.

64. Van Engen A, Lie X, Noren T, Nordling S, Norgaard K. Costeffectiveness of fidaxomicin for the treatment of Clostridium difficile infection (CDI) in Sweden. Value Health. 2014;17(7):A673-4.

65. Varier RU, Biltaji E, Smith KJ, Roberts MS, Jensen MK, LaFleur $\mathrm{J}$, et al. Cost-effectiveness analysis of treatment strategies for initial Clostridium difficile infection. Clin Microbiol Infect. 2014;20(12):1343-51.

66 Watt M, McCrea C, Johal S, Posnett J, Nazir J. A cost-effectiveness and budget impact analysis of fidaxomicin for treating Clostridium difficile patients in Germany. Value Health. 2015;18(3):A233.

67 Surawicz CM, Alexander J. Treatment of refractory and recurrent Clostridium difficile infection. Nat Rev Gastroenterol Hepatol. 2011;8(6):330-9.

68 Sculpher MJ, Pang FS, Manca A, Drummond MF, Golder S, Urdahl $\mathrm{H}$, et al. Generalisability in economic evaluation studies in healthcare: a review and case studies. Health Technol Assess (Winchester, England). 2004;8(49):iii-iv (1-192).

69 Heimann SM, Vehreschild JJ, Cornely OA, Wisplinghoff H, Hallek M, Goldbrunner R, et al. Economic burden of Clostridium difficile associated diarrhoea: a cost-of-illness study from a German tertiary care hospital. Infection. 2015;43(6):707-14.

70 Garey KW, Aitken SL, Gschwind L, Goddu S, Xie Y, Duff C, et al. Development and validation of a Clostridium difficile healthrelated quality-of-life questionnaire. J Clin Gastroenterol. 2016;50(8):631-7.

71 Goldenberg SD, Brown S, Edwards L, Gnanarajah D, Howard P, Jenkins $\mathrm{D}$, et al. The impact of the introduction of fidaxomicin on the management of Clostridium difficile infection in seven NHS secondary care hospitals in England: a series of local service evaluations. Eur J Clin Microbiol Infect Dis. 2016;35(2):251-9.

72 Feher C, Mensa J. A comparison of current guidelines of five international societies on Clostridium difficile infection management. Infect Dis Ther. 2016;5(3):207-30.

73 Marseille E, Larson B, Kazi DS, Kahn JG, Rosen S. Thresholds for the cost-effectiveness of interventions: alternative approaches. Bull World Health Organ. 2015;93(2):118-24. 